\title{
Sistema para la instalación de cubiertas y fachadas cerámicas autoventiladas denominado: SKIN-KER ${ }^{\circledR}$
}

\author{
ALBERTO PUERTA LÓPEZ, ANDRÉS CASANOVA RAMÓN-BORJA
}

TEJAS BORJA S.A.U. Ctra. Llíria a Pedralba Km 3. 46160 Llíria (Valencia).

Este trabajo ha sido premiado con el Alfa de Oro en la Feria Internacional de Cerámica de Valencia CEVISAMA 2012

\begin{abstract}
La Empresa Tejas Borja S.A.U. ha sido galardonada por La Sociedad Española de Cerámica y Vidrio con un Alfa de Oro en su edición de 2012 por el siguiente trabajo de investigación: el desarrollo de un sistema de instalación que permite crear una cubierta autoventilada con piezas de gres porcelánico modificadas, y hacerlo extensivo a la fachada ventilada, permitiendo envolver a toda la vivienda con una misma calidad y estética.

El artículo describe las distintas etapas del proyecto tanto de diseño de los distintos componentes como de verificación mediante simulaciones y ensayos de laboratorio de los distintos elementos que componen el sistema y los materiales auxiliares que intervienen en el montaje.
\end{abstract}

Palabras clave: Tejas Borja, SKIN-KER ${ }^{\oplus}$, cubierta cerámica, teja porcelánica, cubierta autoventilada.

Installation system for ceramic self-ventilated roofs and facades called: SKIN-KER ${ }^{\circledR}$

The Company Tejas Borja S.A.U. has been awarded by the Spanish Ceramic and Glass Society with the 2012 Golden Alfa Pize for its research in the development of an installation system that creates a self-ventilated roof with modified red body porcelain tiles, and make it extended to a ventilated façade, allowing the entire house wrap with the same quality and aesthetics.

The article describes the various stages of the project design both of individual components as well as verification by simulations and lab testing of the different elements of the system and auxiliary materials involved in the installation.

Key words: Tejas Borja, SKIN-KER ${ }^{\circledast}$, ceramic roofs, porcelain roof tile, self-ventilated roof.

\section{INTRODUCCIÓN}

Tejas Borja es una Empresa centenaria, líder en su sector, especializada en el diseño y fabricación de cubiertas cerámicas, con una cuota de mercado nacional cercana al 20\%, una capacidad de producción de más de 100 millones de tejas / año, y una cuota de exportación del $45 \%$ a más de 80 países.

Participa activamente como miembro en la asociación empresarial Hispalyt y como miembro del consejo en el ITC. En los últimos años ha apostado fuertemente por la investigación obteniendo varias patentes en productos de teja cerámica y realizando diferentes proyectos financiados por el Ministerio de Ciencia y Tecnología y CDTI.

El objetivo del presente proyecto, ha sido la obtención de un sistema de cubierta de teja plana de gran formato que sirva de elemento de diseño innovador para la arquitectura vanguardista, que logre integrarse con las nuevas tecnologías de captación de energía, y que se pueda montar de forma modular y rápida, con una reposición fácil y sencilla.

$\mathrm{El}$ proyecto se plantea utilizando los materiales y sistemas de producción existentes en el mercado y que no suponen nuevas inversiones para el sector, obteniendo un producto de dimensión internacional, fácilmente exportable y con todas las posibilidades del diseño actual.
El sistema diseñado resuelve perfectamente la construcción de una cubierta autoventilada con un material de gran calidad, posibilidades de diseño y ligereza, y que se hace extensivo a los paramentos verticales, creando una fachada ventilada con las mismas prestaciones. Del mismo modo al poder utilizar el mismo producto para construir el suelo, podemos crear una piel que puede envolver a toda la vivienda con un mismo material, aumentando las posibilidades de decoración y diseño de la vivienda.

\section{ANTECEDENTES Y ESTADO DE LA TÉCNICA.}

Tradicionalmente las cubiertas cerámicas se han realizado mediante la utilización de tejas conformadas por extrusión y prensado, con unas geometrías que podríamos clasificar en Tejas Curvas, Tejas Planas y Tejas Mixtas. Por término medio las Tejas cerámicas más utilizadas suelen instalar doce piezas por metro cuadrado, con un peso sobre la estructura de 45 kilos por metro instalado.

El diseño de los elementos utilizados en el proceso, prensas, secaderos y hornos junto a la resistencia del propio material, hacen que sea difícil fabricar piezas en formatos más grandes, y con geometrías que se aparten mucho de lo ya existente. 
Esta compleja geometría dificulta además la decoración de las piezas mediante las últimas técnicas desarrolladas para la cerámica plana, limitando la diversidad de las mismas.

Ante los cambios en las últimas décadas en la arquitectura moderna, se demanda cada vez más desde los profesionales implicados en el diseño, la utilización de elementos de construcción con formatos mayores, más planos, adaptados a las nuevas líneas, con mayor posibilidad de decoraciones en colores y texturas, y con una mayor eficiencia energética. Del mismo modo cada vez son más las necesidades funcionales de la cubierta, al adaptar e integrar en la propia cubierta, distintos elementos de captación, instalaciones auxiliares etc., que precisan de acceso y espacio para su instalación.

Al no disponer del material cerámico adecuado a todas estas necesidades estéticas y funcionales desde el sector tradicional de la teja cerámica, se inicia el proyecto de diseñar un sistema que permita construir una cubierta cerámica mediante la utilización de piezas planas de gres porcelánico, modificadas para mejorar sus prestaciones y poderse adaptar a esta nueva funcionalidad.

La técnica se encuadra en el sector de la construcción para recubrimientos de paramentos autoventilados inclinados y verticales. La invención se refiere a la sujeción de piezas planas dedicadas al recubrimiento ornamental y de protección en las cubiertas inclinadas y en fachadas de todo tipo de edificación, favoreciendo la seguridad, la auto-ventilación, la evacuación de aguas pluviales, y facilita la instalación de nuevos materiales y energías renovables.

En la última década se han diseñado numerosos sistemas de fachada ventilada, que se basa en un sistema constructivo de cerramiento exterior constituido por una hoja interior, una capa aislante, y una hoja exterior no estanca. Este tipo de fachada por lo general permite acabados duraderos y de gran calidad, y ofrece buenas prestaciones térmicas. Es una solución habitual en edificios institucionales y representativos.

Sin embargo no se ha desarrollado ningún sistema que se pueda adaptar a la cubierta inclinada, y que permita la utilización de grandes formatos planos, creando una estructura ventilada, que se pueda hacer extensiva a la fachada, y que resuelva todos los encuentros necesarios para la ejecución de una cubierta estanca al agua de lluvia, mediante el desarrollo de las piezas para los remates laterales y de cumbrera.

\section{DESARROLLO DE LA TÉCNICA APLICADA Y REALIZACIONES PRÁCTICAS.}

Las distintas etapas del proyecto han sido las siguientes:

\subsection{Estudio y diseño de los componentes metálicos de sujeción, piezas especiales de remate y materiales auxiliares de cubierta.}

Una vez seleccionado el producto base por sus dimensiones se diseñan los herrajes metálicos de sujeción, anclaje y evacuación que sirven como base para el montaje del sistema tanto en cubierta como en fachada. Los Rastreles en U tienen la función de fijar el sistema al soporte, creando la cámara de ventilación y marcando el paso longitudinal entre las piezas. Sirve de alojamiento para la presilla que sujeta la pieza cerámica en su posición de colocación, y cumple la función de canal de evacuación para el agua que pueda entrar entre las juntas longitudinales.

Tras diseñar el paño principal, se estudian las piezas especiales necesarias para crear el remate lateral y de cumbrera, obteniendo mediante corte y pegado los laterales, caballetes y tapones necesarios para realizar la función de cierre y mantener la estética de la cubierta.

Tanto el diseño individual de cada elemento como la sistemática de colocación se recogen en el manual de instalación creado.

Además de los elementos metálicos y cerámicos, se realiza un estudio de los materiales auxiliares que intervienen en la fijación y aislamiento del sistema sobre la cubierta. Según sus características y durabilidad, se define el tipo de adhesivo químico como un sellador de poliuretano monocomponente

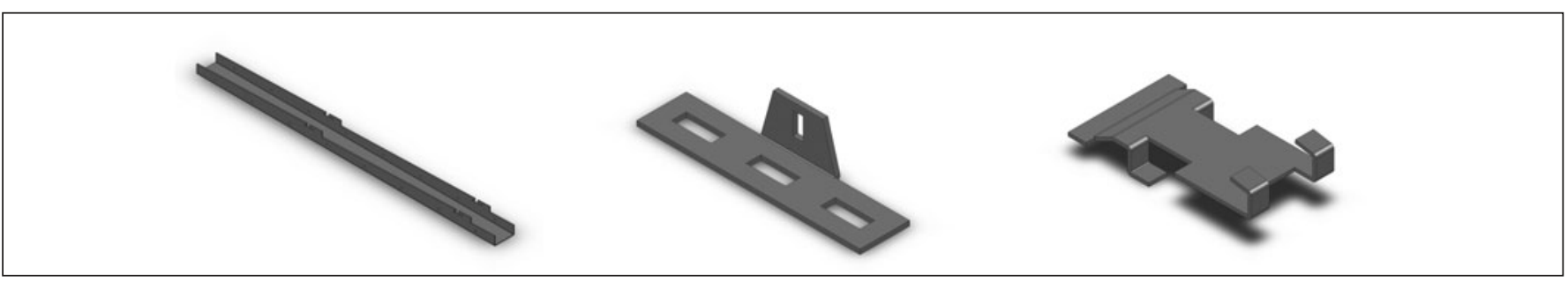

Figura 1. Herrajes metálicos

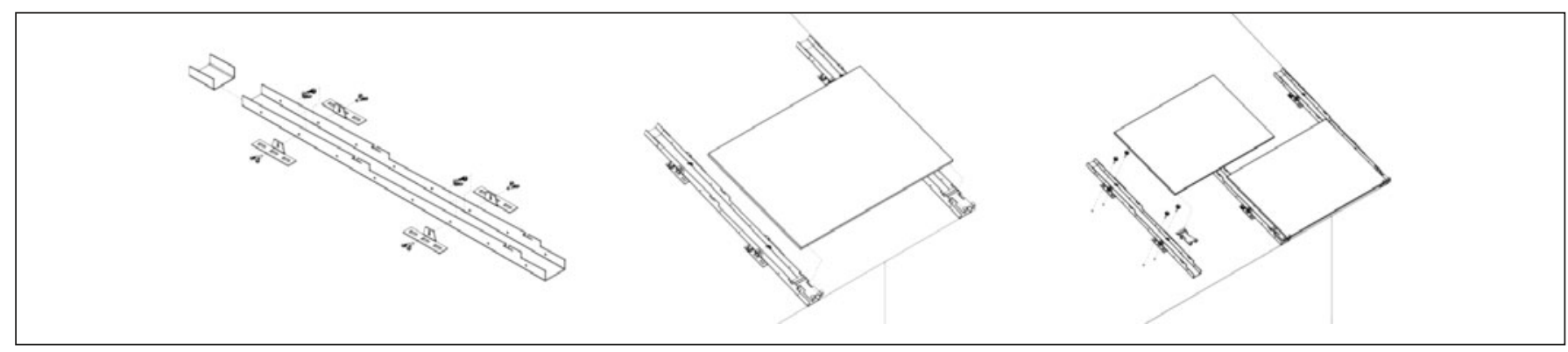

Figura 2. Manual de instalación. 
elástico, que servirá de fijación entre las piezas cerámicas, impedirá el movimiento entre ellas, amortiguará posibles impactos y evitará la entrada de agua por fuerte viento.

Para el montaje de las piezas especiales, se define un tipo de adhesivo bicomponente a base de resinas epoxídicas de gran resistencia química y mecánica.

Para el aislamiento total de la cubierta se definen dos tipos de geomembranas para utilizar en dos rangos distintos de pendientes. Una membrana multicapa impermeable y transpirable de baja densidad para las cubiertas de gran pendiente, y una lámina impermeable de alta densidad para pendientes menores. Ambas cumplen la función de barrera de vapor, impermeabilizan el soporte y evitan las condensaciones por humedad.

\subsection{Construcción estructura piloto para ensayos prácticos de estanqueidad.}

Tras el desarrollo del sistema se realiza un primer paño piloto en el que se comprueba la efectividad del sistema durante la instalación y el remate con las piezas especiales creadas, una vez ejecutado se ensaya su efectividad como cubierta estanca al agua de lluvia, mediante la instalación de un circuito cerrado de agua, que proyecta sobre el paño mediante una bomba y unos aspersores, distintas cantidades de agua simulando la lluvia, controladas mediante un caudalímetro. Del mismo modo se observa la eficacia del sistema en condiciones reales de fuertes lluvias.

\subsection{Modelización, análisis y definición de materiales.}

A partir de esta etapa se inicia la colaboración con el Instituto de Tecnología Cerámica para realizar las comprobaciones teóricas mediante modelización de elementos finitos y ensayos prácticos que nos ayuden a definir las propiedades de los materiales más idóneos para el correcto funcionamiento del sistema, ante los distintos esfuerzos que va a ser sometido durante su uso.

En el modelo 1 se realiza un estudio de carga concentrada, simulando los casos más desfavorables durante su instalación y su uso, mediante la norma UNE-EN 15037-3, que nos lleva a obtener y definir la tensión máxima como función del espesor de la pieza utilizada.

En el modelo 2 se estudia la succión del viento sobre el sistema mediante la los caudales definidos en la Norma americana ASTM C 1569-03, obteniendo el desplazamiento de la teja por efecto de la succión mediante la presión de succión aplicada, el espesor de la presilla, el límite elástico del material y la tensión máxima de la presilla, no observando fallos de sujeción por elevación excesiva ni tensiones que conduzcan al fallo de la del sistema.

\section{Modelo 1 - Carga concentrada}

Tensión máxima como función del espesor

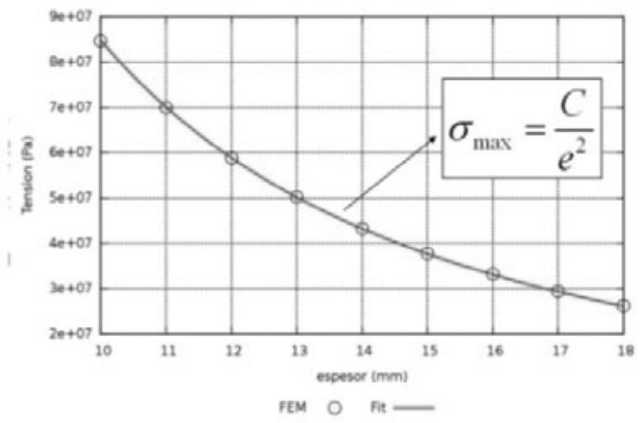

\section{Modelo 1 - Carga concentrada}

Perfiles de tensión entre apoyos

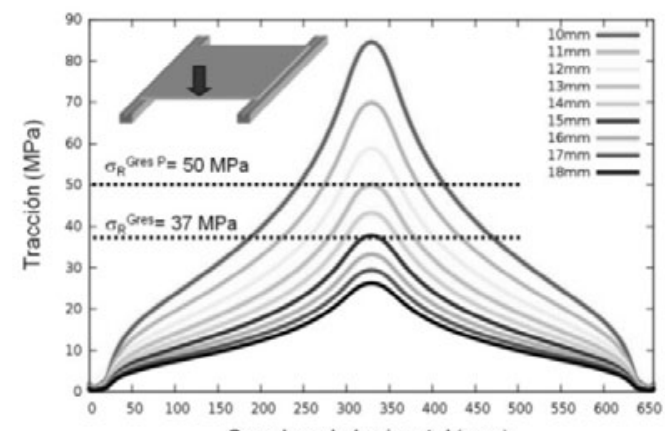

Figura 3. Modelo de carga concentrada.

\section{Modelo 2 - Succión del viento \\ Configuración estudiada}

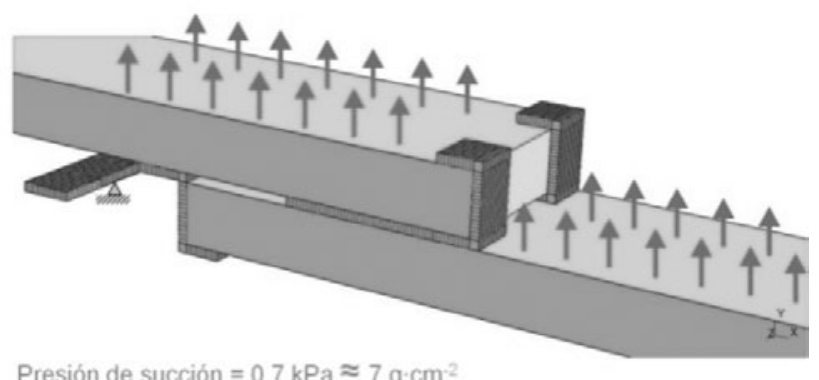

Modelo 2 - Succión del viento

Tensiones en la presilla por efecto de la succión

Tensión de Von Mises

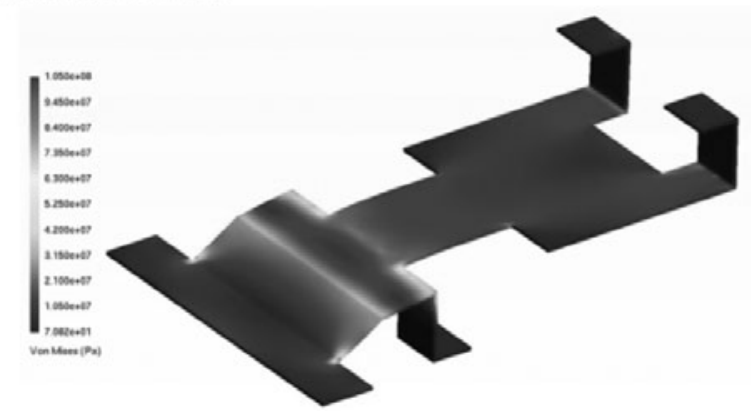

Figura 4. Modelo de succión al viento. 
En el modelo 3 se estudia la carga tangencial tanto en cubierta como en fachada, calculando las tensiones máximas en la presilla mediante la tensión de Von Mises, el cálculo de desplazamientos y la deformación plástica del material, definiendo el tipo de acero y espesor más adecuado para el uso del sistema como cubierta y como fachada, que eviten problemas de desprendimientos de las piezas.

En el modelo 4 se realiza un estudio teórico de cargas, para evaluar la carga crítica del pandeo sobre las alas del rastrel, concluyendo que con la configuración estudiada no se observan problemas de deformación.

Tras la etapa de modelización se inicia la comprobación mediante ensayos de las propiedades del material cerámico que compone la cubierta, para validar los modelos estudiados en cuanto a espesores y resistencias requeridas. Las piezas fueron sometidas a ensayos de resistencia a la flexión y carga de rotura, absorción de agua, resistencia al impacto y a carga concentrada en diferentes posiciones de la pieza. Los resultados obtenidos se muestran en la siguiente tabla.

\section{RESUMEN DE LOS RESULTADOS}

En la tabla siguiente se resumen los resultados obtenidos en los diferentes ensayos realizados:

Muestra 1: TEJADO MADERA (SKIN-KER)

\begin{tabular}{|c|c|c|}
\hline & Ensayo & Resultado \\
\hline \multirow{3}{*}{$\begin{array}{l}\text { Resistencia a la } \\
\text { flexión. Baldosas }\end{array}$} & \multirow{3}{*}{ UNE-EN ISO 10545-4 } & Carga de rotura: $2130 \mathrm{~N}$ \\
\hline & & Fuerza de rotura: $3100 \mathrm{~N}$ \\
\hline & & Resistencia a la flexión: $40 \mathrm{~N} / \mathrm{mm}^{2}$ \\
\hline $\begin{array}{l}\text { Absorción de agua. } \\
\text { Baldosas }\end{array}$ & UNE-EN ISO 10545-3 & Absorción de agua media: $0,6 \%$ \\
\hline $\begin{array}{l}\text { Resistencia a la } \\
\text { flexión. Tejas }\end{array}$ & UNE-EN 538 & Carga de rotura: $\mathbf{2 8 3 5 N}$ \\
\hline $\begin{array}{l}\text { Resistencia al } \\
\text { impacto. Tejas }\end{array}$ & UNE 67-032 & Resultado: SATISFACTORIO \\
\hline \multirow{4}{*}{$\begin{array}{l}\text { Resistencia a } \\
\text { carga concentrada }\end{array}$} & Aplicación en el centro de la pieza & Carga de rotura: $2093 \mathrm{~N}$ \\
\hline & Aplicación en el centro de la arista & Carga de rotura: $1398 \mathrm{~N}$ \\
\hline & $\begin{array}{l}\text { Aplicación en el centro de la arista a } \\
6 \mathrm{~cm} \text { del borde }\end{array}$ & Carga de rotura: $1648 \mathrm{~N}$ \\
\hline & $\begin{array}{l}\text { Aplicación en el centro de la arista } \\
\text { sobre pieza solapada } 6 \mathrm{~cm}\end{array}$ & Carga de rotura: $1982 \mathrm{~N}$ \\
\hline
\end{tabular}
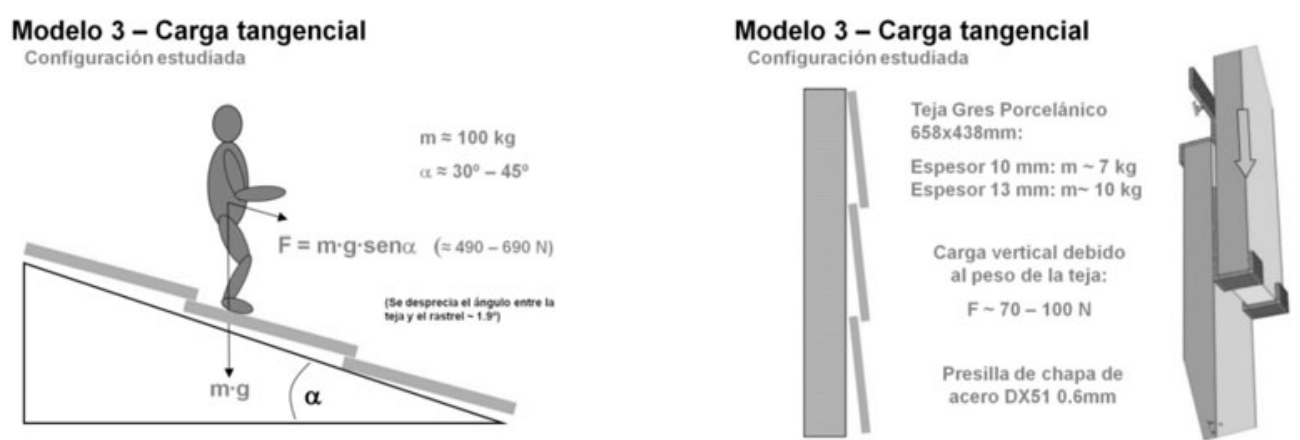

Figura 5. Modelo de carga tangencial en cubierta y fachada.

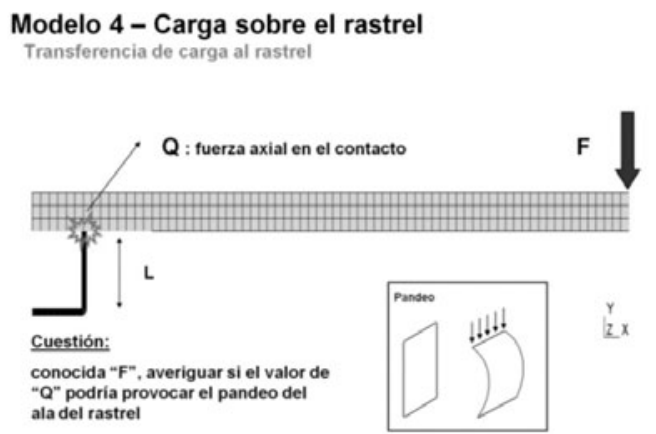

\section{Modelo 4-Carga sobre el rastrel}

Conclusiones

- No se observan problemas de deformación de las alas del rastrel

Sin embargo, esto depende fuertemente de su geometria final:

- L: altura del ala

- e: espesor de la chapa

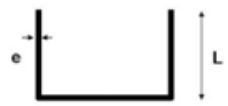

Figura 6. Modelo carga sobre rastrel. 
Con todos los datos obtenidos se crean las especificaciones técnicas de los elementos que componen el sistema. En el material cerámico se especifica la absorción de agua, el espesor y la resistencia mecánica mínima necesaria, junto con las tolerancias dimensionales y deformaciones máximas permitidas. Además se realizan unas modificaciones en el producto de origen, para crear por su cara inferior unas canalizaciones para las posibles condensaciones, y unos galces en todo su perímetro, que permiten el alojamiento de la presilla y crean un cierre longitudinal y un apoyo perfecto en la zona de solape.

En cuanto a los componentes metálicos, se especifican las calidades necesarias para construir cada elemento y los espesores necesarios en cubierta y fachada, así como los medios de anclaje al soporte.

\section{4 Construcción de vivienda real.}

Una vez terminada la etapa de especificación y verificación de materiales se procede a la ejecución de una vivienda real, eligiendo una ubicación con un clima severo que nos sirva para validad el sistema completo. Para ello se elige una vivienda en Tui (Pontevedra), donde durante toda construcción se ha podido evaluar y cuantificar la eficacia y rapidez del sistema constructivo, se han resuelto encuentros y zonas singulares de la cubierta, se ha comprobado el diseño de las piezas especiales de remate, y tras su ejecución se ha podido validar el conjunto de la cubierta, como sistema estanco al agua de lluvia, cubierta autoventilada, y sistema de anclaje al soporte efectivo ante fuertes rachas de viento.

\section{CONCLUSIONES.}

Con el sistema de instalación desarrollado, se logra crear una estructura de fijación resistente a la fuerza del viento, al posible transito sobre ella, y al peso de las piezas cerámicas. La cubierta obtenida es estanca y autoventilada gracias a las canales de evacuación, el remate con piezas especiales y la cámara creada.

Las especificaciones y modificaciones del material cerámico garantizan una óptima resistencia mecánica en todos los supuestos ensayados, una suficiente resistencia al impacto y una comprobada resistencia al hielo.

Los materiales metálicos has sido validados y seleccionados en base a su resistencia y espesor, cumpliendo su función frente a cargas de succión por fuerte viento y a cargas tangenciales en cubierta y fachada, así como las cargas sometidas durante el posible tránsito sobre la cubierta.

Todos los elementos auxiliares que intervienen en la ejecución han sido seleccionados en base a sus propiedades de alta resistencia y durabilidad, que asegurarán su función durante muchos años.

Con el desarrollo realizado se abre un nuevo concepto de cubierta y fachada cerámica, capaz de envolver a toda la vivienda con unas grandes prestaciones técnicas y estéticas.

El sistema utilizado permite obtener una estructura con gran eficiencia energética y ligereza, que favorece la seguridad, la auto-ventilación, la evacuación de aguas pluviales, y que facilita a su vez la instalación de nuevos materiales y energías renovables. La perfección del conjunto la hace adaptable a pendientes de tejado extremas.

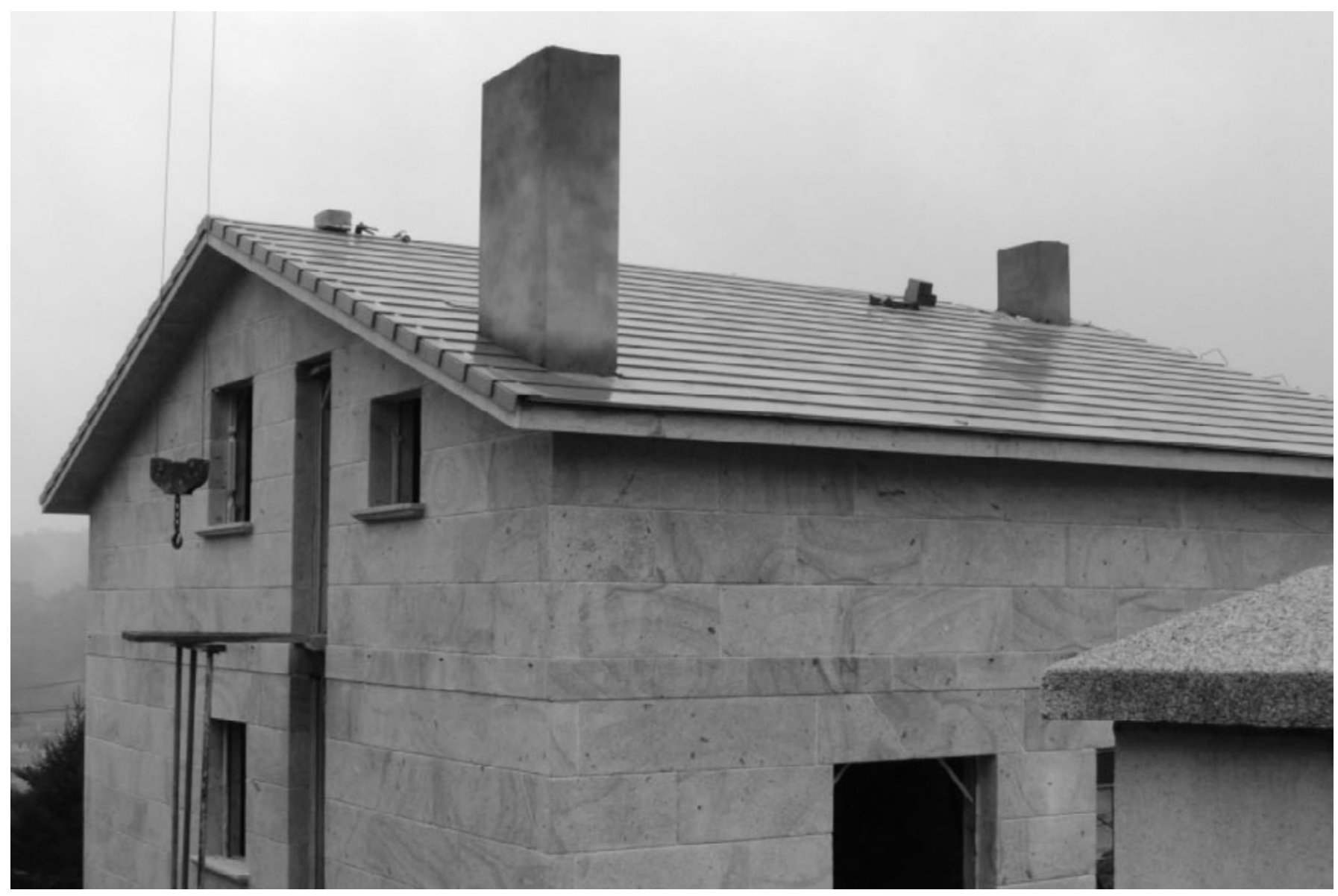


A nivel estético abre nuevas posibilidades al diseño de viviendas, tanto de líneas tradicionales como de líneas más modernas, incorporando al producto cerámico en espacios donde su uso estaba muy limitado. La posibilidad de aplicación de las técnicas más modernas de decoración abre grandes posibilidades al diseño de texturas y acabados, incluida la personalización de la cubierta y la fachada.

En definitiva, este nuevo concepto aporta la posibilidad de utilización de un material con grandes prestaciones como es el gres porcelánico para envolver a la vivienda de una nueva piel, abriendo un nuevo campo al industrial, al arquitecto y al usuario final.

\section{IMPACTO INDUSTRIAL.}

Dada la situación actual del mercado, el sector debe realizar un gran esfuerzo en innovación para poder mantener la situación de liderazgo internacional que tanto esfuerzo ha supuesto conseguir, y que cada día más se ve amenazada por los países emergentes, con menores costes productivos y políticas medio ambientales muy deficientes.

Esta apuesta innovadora, realizada desde una empresa centenaria como Tejas Borja, abre un nuevo espacio donde poder utilizar el material cerámico, donde el producto tradicional no tiene cabida al no adaptarse estéticamente a las nuevas líneas de diseño de viviendas, y donde la cerámica plana no disponía de un sistema que permitiera adaptar el producto a la construcción de cubiertas. Al utilizar sistemas de producción ya existentes, no requiere de nuevas inversiones para el sector, y logra un producto de dimensión internacional, fácilmente exportable.

\section{ENTIDADES IMPLICADAS EN EL DESARROLLO DEL SISTEMA.}

El sistema para la instalación de recubrimientos planos autoventilados verticales o inclinados, nace de la colaboración de diversas empresas e instituciones de investigación.

El proceso, inicialmente desarrollado por la empresa comercial Galigestión S.L encuentra rápidamente el apoyo de Tejas Borja S.A.U, empresa líder en el sector de la teja cerámica, viendo las grandes posibilidades del nuevo sistema y aportando su gran experiencia de más de 110 años como fabricante de cubiertas cerámicas.
Desde Tejas Borja se realiza el proyecto de rediseñar, verificar y validar tanto la calidad de los distintos materiales utilizados como del sistema constructivo en conjunto, para lo cual inicia la colaboración con el Instituto de Tecnología Cerámica a través de su Área de Diseño y Arquitectura Alicer, que goza de una dilatada trayectoria de investigación en desarrollo de productos innovadores y de alto valor añadido, y que aporta su experiencia en el desarrollo de fachadas ventiladas.

Del mismo modo y desde sus inicios se cuenta con la colaboración de la empresa Materiales Essaco S.L y Gutterkel en el desarrollo de los herrajes metálicos y con Cerámicas Realonda S.A en la adaptación y producción del gres porcelánico SKIN-KER.

Todas las empresas que han participado en el proyecto poseen un capital $100 \%$ nacional, aportando desde el punto de vista del impacto social un aspecto positivo, y una posibilidad de negocio que puede beneficiar a la competitividad de todo el sector.

El sistema para la instalación de recubrimientos planos autoventilados verticales o inclinados está en proceso de patente como Modelo de Utilidad $n^{\circ}$ U 201100059, así mismo la denominación comercial del sistema SKIN-KER está registrado como Marca Comunitaria MC 010523074.

\section{BIBLIOGRAFÍA}

(1) Norma UNE-EN ISO 10545-4: 1997 “Baldosas Cerámicas - Parte 4: Determinación de la resistencia a la flexión y de la carga de rotura".

(2) Norma UNE-EN ISO 10545-3:1997 "Baldosas Cerámicas - Parte 3: Determinación de la absorción de agua, de la porosidad abierta, de la densidad relativa aparente, y de la densidad aparente".

(3) Norma UNE-EN 538: 1995 “Tejas de arcilla cocida. Ensayo de resistencia a la flexión".

(4) Norma UNE 67-032: 1985 “Tejas de arcilla cocida. Ensayo de resistencia al impacto".

(5) Norma UNE-EN 15037-3 Apartado 5.2 "Bovedillas de arcilla cocida. Resistencia mecánica. Resistencia al punzonamiento".

(6) Norma UNE-EN 12825: 2002 Apartado 5.5.2 "Pavimentos elevados registrables. Impacto de un cuerpo blando".

(7) ETAG 034 - Edición Abril 2009 "Guideline for European Technical Approval of kits for external wall claddings Part 1: Ventilated cladding elements and associated fixing devices".

(8) Análisis de la intensidad de precipitación: Método de la intensidad contigua. Autor Roberto Moncho Agud.

(9) Cálculo aproximado de la altura de inundación. F. Javier Sánchez San Román---- Dpto. Geología Univ. Salamanca. 